Acta Crystallographica Section E

Structure Reports

Online

ISSN 1600-5368

\section{4-[(E)-(4-Ethoxyphenyl)iminomethyl]- phenol}

\section{Aliakbar Dehno Khalaji, ${ }^{a}$ Karla Fejfarováb ${ }^{\text {* }}$ and Michal Dušek $^{b}$}

a Department of Chemistry, Faculty of Science, Golestan University, Gorgan, Iran, and ${ }^{\mathbf{b}}$ Institute of Physics ASCR, v.v.i., Na Slovance 2, 18221 Praha 8, Czech Republic

Correspondence e-mail: fejfarov@fzu.cz

Received 31 July 2012; accepted 1 August 2012

Key indicators: single-crystal X-ray study; $T=120 \mathrm{~K}$; mean $\sigma(\mathrm{C}-\mathrm{C})=0.002 \AA$; $R$ factor $=0.031 ; w R$ factor $=0.103 ;$ data-to-parameter ratio $=11.9$.

In the title compound, $\mathrm{C}_{15} \mathrm{H}_{15} \mathrm{NO}_{2}$, the dihedral angle between the benzene rings is $52.04(5)^{\circ}$ and the molecule has an $E$ conformation about the central $\mathrm{C}=\mathrm{N}$ bond. In the crystal, molecules are connected by $\mathrm{O}-\mathrm{H} \cdots \mathrm{N}$ hydrogen bonds, forming zigzag chains along the $b$ axis. The crystal packing also features weak $\mathrm{C}-\mathrm{H}$... O interactions.

\section{Related literature}

For Schiff base derivatives and related structures, see: Fejfarová et al. (2010); Özek et al. (2010); Akkurt et al. (2008); Khalaji et al. (2008, 2009) For applications and properties of Schiff bases, see: Dalapati et al. (2011); Keypour et al. (2010); Khalil et al. (2009); Khanmohammadi et al. (2009); Sun et al. (2012); Da Silva et al. (2011).<smiles>CCOc1ccc(/N=C/c2ccc(O)cc2)cc1</smiles>

\section{Experimental}

Crystal data
$\mathrm{C}_{15} \mathrm{H}_{15} \mathrm{NO}_{2}$

$M_{r}=241.3$

Orthorhombic, $\mathrm{Pbca}$

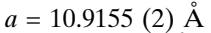

$b=9.4056$ (2) $\AA$

$c=25.0422(5) \AA$
$V=2571.00(9) \AA^{3}$

$Z=8$

$\mathrm{Cu} K \alpha$ radiation

$\mu=0.67 \mathrm{~mm}^{-1}$

$T=120 \mathrm{~K}$

$0.54 \times 0.20 \times 0.03 \mathrm{~mm}$
Data collection

Agilent Xcalibur diffractometer with an Atlas (Gemini ultra $\mathrm{Cu}$ ) detector

Absorption correction: multi-scan (CrysAlis PRO; Agilent, 2011)

$T_{\min }=0.795, T_{\max }=1$

Refinement

$R\left[F^{2}>3 \sigma\left(F^{2}\right)\right]=0.031$

$w R\left(F^{2}\right)=0.103$

$S=1.71$

1978 reflections

166 parameters

22644 measured reflections 1978 independent reflections $R_{\text {int }}=0.028$

$\theta_{\max }=61.1^{\circ}$

$\mathrm{H}$ atoms treated by a mixture of independent and constrained refinement

$\Delta \rho_{\max }=0.11{\mathrm{e} \AA^{-3}}^{-3}$

$\Delta \rho_{\min }=-0.11 \mathrm{e} \AA^{-3}$ 1780 reflections with $I>3 \sigma(I)$

Table 1

Hydrogen-bond geometry $\left(\AA{ }^{\circ}\right)$.

\begin{tabular}{lllll}
\hline$D-\mathrm{H} \cdots A$ & $D-\mathrm{H}$ & $\mathrm{H} \cdots A$ & $D \cdots A$ & $D-\mathrm{H} \cdots A$ \\
\hline $\mathrm{C} 7-\mathrm{H} 7 b \cdots \mathrm{O} 2^{\mathrm{i}}$ & 0.96 & 2.50 & $3.3393(15)$ & 147 \\
$\mathrm{O} 2-\mathrm{H} 2 o \cdots \mathrm{N} 1^{\mathrm{ii}}$ & $0.894(17)$ & $1.825(17)$ & $2.7098(12)$ & $170.0(14)$ \\
\hline
\end{tabular}

Symmetry codes: (i) $x-\frac{1}{2}, y+1,-z+\frac{3}{2}$; (ii) $-x+1, y-\frac{1}{2},-z+\frac{3}{2}$.

Data collection: CrysAlis PRO (Agilent, 2011); cell refinement: CrysAlis PRO; data reduction: CrysAlis PRO; program(s) used to solve structure: SIR2002 (Burla et al., 2003); program(s) used to refine structure: JANA2006 (Petříček et al., 2006); molecular graphics: DIAMOND (Brandenburg \& Putz, 2005); software used to prepare material for publication: $J A N A 2006$.

We acknowledge Golestan University for partial support of this work, the Institutional Research Plan No. AVOZ10100521 of the Institute of Physics and the Praemium Academiae Project of the Academy of Sciences of the Czech Republic.

Supplementary data and figures for this paper are available from the IUCr electronic archives (Reference: BT5991).

\section{References}

Agilent (2011). CrysAlis PRO. Agilent Technologies, Yarnton, England.

Akkurt, M., Jarrahpour, A., Aye, M., Gençaslan, M. \& Büyükgüngör, O. (2008). Acta Cryst. E64, o2087.

Brandenburg, K. \& Putz, H. (2005). DIAMOND. Crystal Impact GbR, Bonn, Germany.

Burla, M. C., Camalli, M., Carrozzini, B., Cascarano, G. L., Giacovazzo, C., Polidori, G. \& Spagna, R. (2003). J. Appl. Cryst. 36, 1103.

Dalapati, S., Alam, M. A., Jana, S. \& Guchhait, N. (2011). J. Fluorine Chem. 132, 536-540.

Da Silva, C. M., Da Silva, D. L., Modolo, L. V., Alves, R. B., de Resende, M., Martins, C. V. B. \& de Fatima, A. (2011). J. Adv. Res. 2, 1-8.

Fejfarová, K., Khalaji, A. D. \& Dušek, M. (2010). Acta Cryst. E66, o2874.

Keypour, H., Dehghani-Firouzabadi, A. A., Rezaeivala, M. \& Goudarziafshar, H. (2010). J. Iran. Chem. Soc. 7, 820-824.

Khalaji, A. D. \& Harrison, W. T. A. (2008). Anal. Sci. 24, x3-x4.

Khalaji, A. D., Weil, M., Gotoh, K. \& Ishida, H. (2009). Acta Cryst. E65, o436.

Khalil, R.A., Jalil, A.H. \& Abd-Alrazzak, A. Y. (2009). J. Iran. Chem. Soc. 6, 345-352.

Khanmohammadi, H., Salehifard, M. \& Abnosi, M. H. (2009). J. Iran. Chem. Soc. 6, 300-309.

Özek, A., Koşar, B., Albayrak, Ç. \& Büyükgüngör, O. (2010). Acta Cryst. E66, 0684.

Petříček, V., Dušek, M. \& Palatinus, L. (2006). JANA2006. Institute of Physics, Praha, Czech Republic.

Sun, Y., Wang, Y., Liu, Z., Huang, C. \& Yu, C. (2012). Spectrochim. Acta Part $A$, 96, 42-50. 


\section{supporting information}

Acta Cryst. (2012). E68, o2646 [doi:10.1107/S1600536812034253]

\section{4-[(E)-(4-Ethoxyphenyl)iminomethyl]phenol}

\section{Aliakbar Dehno Khalaji, Karla Fejfarová and Michal Dušek}

\section{S1. Comment}

Schiff base compounds exhibit a broad range of biological activities, including antifungal, and antibacterial (Da Silva et al., 2011). They are used as anion sensors (Dalapati et al., 2011; Khalil et al., 2009), non-linear optics compounds (Sun et al., 2012), and as versatile ligands in coordination chemistry (Khanmohammadi et al., 2009; Keypour et al., 2010). The present work is part of a structural study of Schiff bases (Khalaji et al., 2008, 2009; Fejfarová et al., 2010) and we report here the structure of (E)-(4-hydroxybenzylidene)-4-ethoxyaniline, (1).

The molecule of (1) (Fig. 1) has an $E$ conformation about the central $\mathrm{C}=\mathrm{N}$ bond and the $\mathrm{C}=\mathrm{N}$ and $\mathrm{C}-\mathrm{N}$ bond lengths of 1.2853 (45) and 1.4250 (14) Å agree well with the corresponding distances in other Schiff bases (Akkurt et al., 2008; Özek et al., 2010; Khalaji et al., 2008, 2009; Fejfarová et al., 2010). The dihedral angle between the two benzene rings is $52.04(5)^{\circ}$. The ethoxy group is almost coplanar with the adjacent ring [dihedral angle $\left.3.51(12)^{\circ}\right]^{\text {. The molecules are }}$ connected by intermolecular $\mathrm{O}-\mathrm{H} \cdots \mathrm{N}$ hydrogen bonds, forming zigzag chains along the $b$ axis (Fig. 2). The crystal structure is further stabilized by intermolecular $\mathrm{C}-\mathrm{H} \cdots \mathrm{O}$ hydrogen bonds.

\section{S2. Experimental}

To a stirring solution of the 4-hydroxybenzaldehyde $(0.2 \mathrm{mmol}$, in $5 \mathrm{ml}$ of methanol) was added 4-ethoxyaniline $(0.2$ $\mathrm{mmol}$ ) in $10 \mathrm{ml}$ of methanol and the mixture was stirred for $1 \mathrm{~h}$ in air at $323 \mathrm{~K}$ and was then left at room temperature for several days without disturbance yielding suitable crystals of 1 that subsequently were filtered off and washed with Et2O. Yield: $82 \%$

\section{S3. Refinement}

The hydroxyl hydrogen atom was found in difference Fourier maps and its coordinates were refined. All other hydrogen atoms were calculated geometrically and refined as riding on their parent atoms. The methyl $\mathrm{H}$ atoms were allowed to rotate freely about the adjacent $\mathrm{C}-\mathrm{C}$ bond. The displacement coefficients of hydrogen atoms $U_{\text {iso }}(\mathrm{H})$ were set to $1.5 U_{\text {eq }}(\mathrm{C}$, O) for the methyl- and hydroxyl- groups and to to $1.2 U_{\mathrm{eq}}(\mathrm{C})$ for the $\mathrm{CH}$ - and $\mathrm{CH} 2$ - groups. 


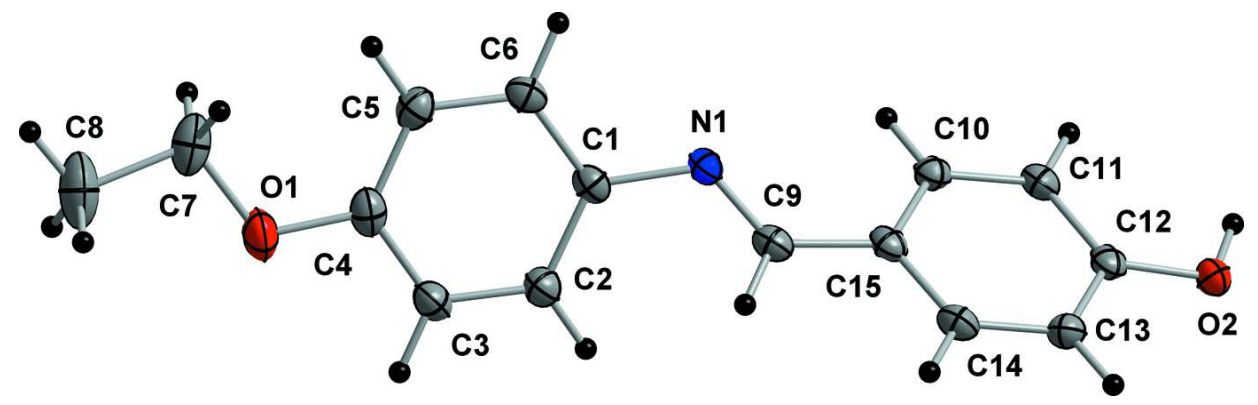

\section{Figure 1}

Molecular structure of the title compound. Displacement ellipsoids are shown at the $50 \%$ probability level.

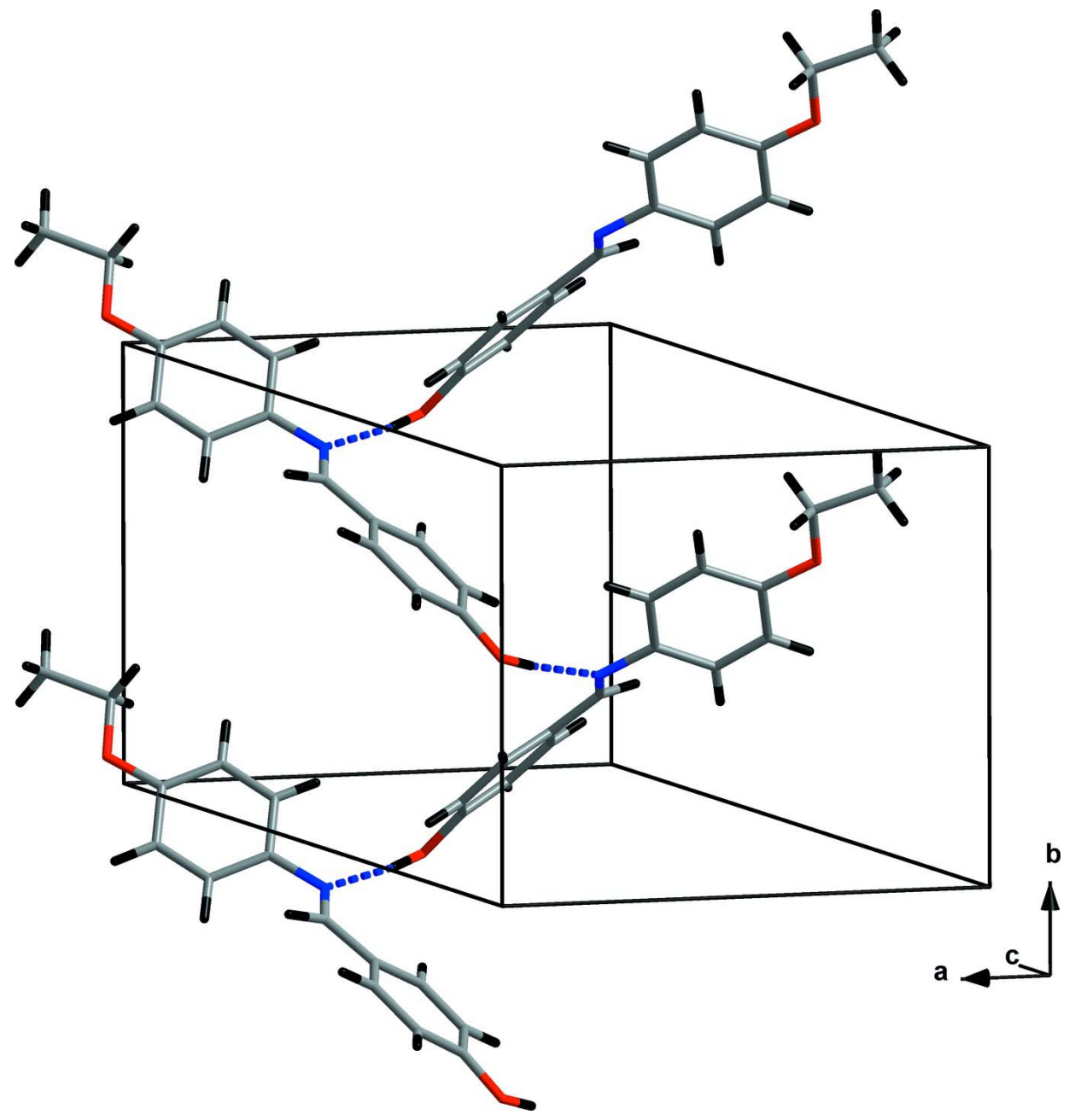

Figure 2

Hydrogen-bonded chain propagating along the $b$ axis. Hydrogen bonds are drawn as dashed lines.

\section{4-[(E)-(4-Ethoxyphenyl)iminomethyl]phenol}

Crystal data

$\mathrm{C}_{15} \mathrm{H}_{15} \mathrm{NO}_{2}$

$M_{r}=241.3$

Orthorhombic, $\mathrm{Pbca}$

Hall symbol: -P 2ac 2ab 
$a=10.9155$ (2) $\AA$

$b=9.4056(2) \AA$

$c=25.0422(5) \AA$

$V=2571.00(9) \AA^{3}$

$Z=8$

$F(000)=1024$

$D_{\mathrm{x}}=1.246 \mathrm{Mg} \mathrm{m}^{-3}$

\section{Data collection}

Agilent Xcalibur

diffractometer with an Atlas (Gemini ultra $\mathrm{Cu}$ ) detector

Radiation source: Enhance Ultra (Cu) X-ray

Source

Mirror monochromator

Detector resolution: 10.3784 pixels $\mathrm{mm}^{-1}$

Rotation method data acquisition using $\omega$ scans

Absorption correction: multi-scan

(CrysAlis PRO; Agilent, 2011)

Refinement

Refinement on $F^{2}$

$R\left[F^{2}>2 \sigma\left(F^{2}\right)\right]=0.031$

$w R\left(F^{2}\right)=0.103$

$S=1.71$

1978 reflections

166 parameters

0 restraints

57 constraints

Special details

Experimental. Absorption correction: CrysAlis PRO (Agilent, 2011). Empirical absorption correction using spherical harmonics, implemented in SCALE3 ABSPACK scaling algorithm.

Refinement. The refinement was carried out against all reflections. The conventional $R$-factor is always based on $F$. The goodness of fit as well as the weighted $R$-factor are based on $F$ and $F^{2}$ for refinement carried out on $F$ and $F^{2}$, respectively. The threshold expression is used only for calculating $R$-factors etc. and it is not relevant to the choice of reflections for refinement.

The program used for refinement, Jana2006, uses the weighting scheme based on the experimental expectations, see _refine_ls_weighting_details, that does not force $S$ to be one. Therefore the values of $S$ are usually larger than the ones from the SHELX program.

Fractional atomic coordinates and isotropic or equivalent isotropic displacement parameters $\left(\AA^{2}\right)$

\begin{tabular}{lllll}
\hline & $x$ & $y$ & $z$ & $U_{\text {iso }} * / U_{\text {eq }}$ \\
\hline O1 & $-0.08099(8)$ & $0.58106(10)$ & $0.55939(4)$ & $0.0344(3)$ \\
O2 & $0.50129(8)$ & $-0.13939(9)$ & $0.85269(3)$ & $0.0251(3)$ \\
N1 & $0.26997(8)$ & $0.30259(10)$ & $0.68326(4)$ & $0.0196(3)$ \\
C1 & $0.18053(10)$ & $0.37189(12)$ & $0.65100(4)$ & $0.0200(3)$ \\
C2 & $0.06035(11)$ & $0.32217(13)$ & $0.64660(4)$ & $0.0234(4)$ \\
C3 & $-0.02398(11)$ & $0.39425(13)$ & $0.61577(5)$ & $0.0260(4)$ \\
C4 & $0.00974(12)$ & $0.51683(14)$ & $0.58837(4)$ & $0.0259(4)$ \\
C5 & $0.12999(11)$ & $0.56466(13)$ & $0.59109(5)$ & $0.0251(4)$ \\
C6 & $0.21487(11)$ & $0.49095(12)$ & $0.62174(5)$ & $0.0228(4)$
\end{tabular}

$\mathrm{Cu} K \alpha$ radiation, $\lambda=1.5418 \AA$

Cell parameters from 14821 reflections

$\mu=0.67 \mathrm{~mm}^{-1}$

$T=120 \mathrm{~K}$

Plate, light yellow

$0.54 \times 0.20 \times 0.03 \mathrm{~mm}$

$T_{\min }=0.795, T_{\max }=1$

22644 measured reflections

1978 independent reflections

$R_{\text {int }}=0.028$

$\theta_{\max }=61.1^{\circ}, \theta_{\min }=3.5^{\circ}$

$h=-12 \rightarrow 12$

$k=-10 \rightarrow 10$

$l=-28 \rightarrow 27$

$\mathrm{H}$ atoms treated by a mixture of independent

and constrained refinement

Weighting scheme based on measured s.u.'s $w=$

$(\Delta / \sigma)_{\max }=0.006$

$\Delta \rho_{\max }=0.11$ e $\AA^{-3}$

$\Delta \rho_{\min }=-0.11$ e $\AA^{-3}$ 
supporting information

$\begin{array}{lllll}\text { C7 } & -0.04877(15) & 0.70560(14) & 0.52934(5) & 0.0357(4) \\ \text { C8 } & -0.16378(16) & 0.75963(18) & 0.50327(7) & 0.0509(5) \\ \text { C9 } & 0.23493(11) & 0.24457(11) & 0.72715(5) & 0.0208(4) \\ \text { C10 } & 0.42099(10) & 0.09397(12) & 0.74119(5) & 0.0198(4) \\ \text { C11 } & 0.48630(10) & -0.00196(12) & 0.77159(4) & 0.0199(4) \\ \text { C12 } & 0.44379(10) & -0.04176(12) & 0.82220(4) & 0.0205(4) \\ \text { C13 } & 0.33635(10) & 0.01912(12) & 0.84201(5) & 0.0230(4) \\ \text { C14 } & 0.27084(11) & 0.11386(12) & 0.81106(4) & 0.0228(4) \\ \text { C15 } & 0.31179(10) & 0.15335(12) & 0.76005(4) & 0.0202(4) \\ \text { H2 } & 0.036296 & 0.23732 & 0.665154 & 0.028^{*} \\ \text { H3 } & -0.106486 & 0.35958 & 0.613196 & 0.0313^{*} \\ \text { H5 } & 0.154298 & 0.648332 & 0.571861 & 0.0301^{*} \\ \text { H6 } & 0.298437 & 0.52267 & 0.622731 & 0.0273^{*} \\ \text { H7a } & 0.009871 & 0.680691 & 0.502344 & 0.0429^{*} \\ \text { H7b } & -0.017107 & 0.776855 & 0.553066 & 0.0429^{*} \\ \text { H8a } & -0.144624 & 0.840538 & 0.481436 & 0.0764^{*} \\ \text { H8b } & -0.198521 & 0.686045 & 0.48144 & 0.0764^{*} \\ \text { H8c } & -0.221636 & 0.786824 & 0.530273 & 0.0764^{*} \\ \text { H9 } & 0.152732 & 0.262562 & 0.738996 & 0.0249^{*} \\ \text { H10 } & 0.450945 & 0.120386 & 0.706538 & 0.0237^{*} \\ \text { H11 } & 0.561133 & -0.041581 & 0.758022 & 0.0239^{*} \\ \text { H13 } & 0.308026 & -0.0049 & 0.877165 & 0.0276^{*} \\ \text { H14 } & 0.196003 & 0.153391 & 0.82466 & 0.0273^{*} \\ \text { H2o } & 0.5725(16) & -0.1625(16) & 0.8374(6) & 0.0377^{*} \\ & & & & \end{array}$

Atomic displacement parameters $\left(\AA^{2}\right)$

\begin{tabular}{lllllll}
\hline & $U^{11}$ & $U^{22}$ & $U^{33}$ & $U^{12}$ & $U^{13}$ & $U^{23}$ \\
\hline O1 & $0.0303(5)$ & $0.0399(6)$ & $0.0330(5)$ & $0.0097(4)$ & $-0.0083(4)$ & $0.0063(4)$ \\
O2 & $0.0188(5)$ & $0.0286(5)$ & $0.0279(5)$ & $0.0040(3)$ & $0.0011(3)$ & $0.0074(3)$ \\
N1 & $0.0168(5)$ & $0.0182(5)$ & $0.0238(5)$ & $-0.0005(4)$ & $-0.0022(4)$ & $-0.0012(4)$ \\
C1 & $0.0181(6)$ & $0.0208(6)$ & $0.0210(6)$ & $0.0025(4)$ & $0.0003(4)$ & $-0.0033(5)$ \\
C2 & $0.0218(6)$ & $0.0235(7)$ & $0.0248(6)$ & $-0.0005(5)$ & $-0.0009(5)$ & $-0.0002(5)$ \\
C3 & $0.0180(6)$ & $0.0325(7)$ & $0.0277(7)$ & $0.0002(5)$ & $-0.0020(5)$ & $-0.0016(5)$ \\
C4 & $0.0263(7)$ & $0.0303(7)$ & $0.0212(6)$ & $0.0081(5)$ & $-0.0028(5)$ & $-0.0019(5)$ \\
C5 & $0.0281(7)$ & $0.0243(6)$ & $0.0229(6)$ & $0.0017(5)$ & $0.0022(5)$ & $0.0005(5)$ \\
C6 & $0.0204(6)$ & $0.0238(6)$ & $0.0241(6)$ & $-0.0003(5)$ & $0.0014(5)$ & $-0.0020(5)$ \\
C7 & $0.0496(9)$ & $0.0313(7)$ & $0.0262(7)$ & $0.0121(6)$ & $-0.0067(6)$ & $0.0008(5)$ \\
C8 & $0.0644(11)$ & $0.0465(9)$ & $0.0418(9)$ & $0.0231(8)$ & $-0.0190(8)$ & $-0.0003(7)$ \\
C9 & $0.0166(6)$ & $0.0199(6)$ & $0.0259(7)$ & $-0.0002(4)$ & $-0.0001(5)$ & $-0.0030(5)$ \\
C10 & $0.0176(6)$ & $0.0207(6)$ & $0.0209(6)$ & $-0.0030(4)$ & $-0.0007(4)$ & $-0.0011(4)$ \\
C11 & $0.0154(6)$ & $0.0205(6)$ & $0.0239(6)$ & $-0.0006(4)$ & $-0.0010(4)$ & $-0.0023(5)$ \\
C12 & $0.0169(6)$ & $0.0196(6)$ & $0.0249(6)$ & $-0.0035(4)$ & $-0.0041(4)$ & $-0.0005(5)$ \\
C13 & $0.0190(6)$ & $0.0271(7)$ & $0.0228(6)$ & $-0.0026(5)$ & $0.0014(5)$ & $0.0015(5)$ \\
C14 & $0.0167(6)$ & $0.0248(6)$ & $0.0267(6)$ & $0.0005(5)$ & $0.0007(5)$ & $-0.0014(5)$ \\
C15 & $0.0171(6)$ & $0.0193(6)$ & $0.0243(6)$ & $-0.0030(4)$ & $-0.0026(5)$ & $-0.0025(5)$ \\
& & & & & & \\
\hline
\end{tabular}


Geometric parameters $\left(\AA,{ }^{\circ}\right)$

\begin{tabular}{|c|c|c|c|}
\hline $\mathrm{O} 1-\mathrm{C} 4$ & $1.3684(15)$ & $\mathrm{C} 7-\mathrm{H} 7 \mathrm{a}$ & 0.96 \\
\hline $\mathrm{O} 1-\mathrm{C} 7$ & $1.4360(16)$ & $\mathrm{C} 7-\mathrm{H} 7 \mathrm{~b}$ & 0.96 \\
\hline $\mathrm{O} 2-\mathrm{C} 12$ & $1.3492(14)$ & $\mathrm{C} 8-\mathrm{H} 8 \mathrm{a}$ & 0.96 \\
\hline $\mathrm{O} 2-\mathrm{H} 2 \mathrm{O}$ & $0.894(17)$ & $\mathrm{C} 8-\mathrm{H} 8 \mathrm{~b}$ & 0.96 \\
\hline $\mathrm{N} 1-\mathrm{C} 1$ & $1.4250(14)$ & $\mathrm{C} 8-\mathrm{H} 8 \mathrm{c}$ & 0.96 \\
\hline $\mathrm{N} 1-\mathrm{C} 9$ & $1.2853(14)$ & $\mathrm{C} 9-\mathrm{C} 15$ & $1.4557(16)$ \\
\hline $\mathrm{C} 1-\mathrm{C} 2$ & $1.3971(16)$ & $\mathrm{C} 9-\mathrm{H} 9$ & 0.96 \\
\hline $\mathrm{C} 1-\mathrm{C} 6$ & $1.3897(16)$ & $\mathrm{C} 10-\mathrm{C} 11$ & $1.3791(16)$ \\
\hline $\mathrm{C} 2-\mathrm{C} 3$ & $1.3795(17)$ & $\mathrm{C} 10-\mathrm{C} 15$ & $1.3985(15)$ \\
\hline $\mathrm{C} 2-\mathrm{H} 2$ & 0.96 & $\mathrm{C} 10-\mathrm{H} 10$ & 0.96 \\
\hline $\mathrm{C} 3-\mathrm{C} 4$ & $1.3913(17)$ & $\mathrm{C} 11-\mathrm{C} 12$ & $1.4006(15)$ \\
\hline $\mathrm{C} 3-\mathrm{H} 3$ & 0.96 & $\mathrm{C} 11-\mathrm{H} 11$ & 0.96 \\
\hline $\mathrm{C} 4-\mathrm{C} 5$ & $1.3892(17)$ & $\mathrm{C} 12-\mathrm{C} 13$ & $1.3961(16)$ \\
\hline $\mathrm{C} 5-\mathrm{C} 6$ & $1.3887(16)$ & $\mathrm{C} 13-\mathrm{C} 14$ & $1.3807(16)$ \\
\hline $\mathrm{C} 5-\mathrm{H} 5$ & 0.96 & $\mathrm{C} 13-\mathrm{H} 13$ & 0.96 \\
\hline $\mathrm{C} 6-\mathrm{H} 6$ & 0.96 & $\mathrm{C} 14-\mathrm{C} 15$ & $1.4033(16)$ \\
\hline $\mathrm{C} 7-\mathrm{C} 8$ & $1.504(2)$ & $\mathrm{C} 14-\mathrm{H} 14$ & 0.96 \\
\hline $\mathrm{C} 4-\mathrm{O} 1-\mathrm{C} 7$ & $117.43(10)$ & $\mathrm{C} 7-\mathrm{C} 8-\mathrm{H} 8 \mathrm{a}$ & 109.47 \\
\hline $\mathrm{C} 12-\mathrm{O} 2-\mathrm{H} 2 \mathrm{o}$ & $109.1(9)$ & $\mathrm{C} 7-\mathrm{C} 8-\mathrm{H} 8 \mathrm{~b}$ & 109.47 \\
\hline $\mathrm{C} 1-\mathrm{N} 1-\mathrm{C} 9$ & $118.37(9)$ & $\mathrm{C} 7-\mathrm{C} 8-\mathrm{H} 8 \mathrm{c}$ & 109.47 \\
\hline $\mathrm{N} 1-\mathrm{C} 1-\mathrm{C} 2$ & $122.33(10)$ & $\mathrm{H} 8 \mathrm{a}-\mathrm{C} 8-\mathrm{H} 8 \mathrm{~b}$ & 109.47 \\
\hline $\mathrm{N} 1-\mathrm{C} 1-\mathrm{C} 6$ & $118.86(10)$ & $\mathrm{H} 8 \mathrm{a}-\mathrm{C} 8-\mathrm{H} 8 \mathrm{c}$ & 109.47 \\
\hline $\mathrm{C} 2-\mathrm{C} 1-\mathrm{C} 6$ & $118.78(10)$ & $\mathrm{H} 8 \mathrm{~b}-\mathrm{C} 8-\mathrm{H} 8 \mathrm{c}$ & 109.47 \\
\hline $\mathrm{C} 1-\mathrm{C} 2-\mathrm{C} 3$ & $120.40(11)$ & $\mathrm{N} 1-\mathrm{C} 9-\mathrm{C} 15$ & $124.24(10)$ \\
\hline $\mathrm{C} 1-\mathrm{C} 2-\mathrm{H} 2$ & 119.8 & $\mathrm{~N} 1-\mathrm{C} 9-\mathrm{H} 9$ & 117.88 \\
\hline $\mathrm{C} 3-\mathrm{C} 2-\mathrm{H} 2$ & 119.8 & $\mathrm{C} 15-\mathrm{C} 9-\mathrm{H} 9$ & 117.88 \\
\hline $\mathrm{C} 2-\mathrm{C} 3-\mathrm{C} 4$ & $120.45(11)$ & $\mathrm{C} 11-\mathrm{C} 10-\mathrm{C} 15$ & $121.02(10)$ \\
\hline $\mathrm{C} 2-\mathrm{C} 3-\mathrm{H} 3$ & 119.77 & $\mathrm{C} 11-\mathrm{C} 10-\mathrm{H} 10$ & 119.49 \\
\hline $\mathrm{C} 4-\mathrm{C} 3-\mathrm{H} 3$ & 119.77 & $\mathrm{C} 15-\mathrm{C} 10-\mathrm{H} 10$ & 119.49 \\
\hline $\mathrm{O} 1-\mathrm{C} 4-\mathrm{C} 3$ & $115.84(11)$ & $\mathrm{C} 10-\mathrm{C} 11-\mathrm{C} 12$ & $120.21(10)$ \\
\hline $\mathrm{O} 1-\mathrm{C} 4-\mathrm{C} 5$ & $124.54(11)$ & $\mathrm{C} 10-\mathrm{C} 11-\mathrm{H} 11$ & 119.89 \\
\hline $\mathrm{C} 3-\mathrm{C} 4-\mathrm{C} 5$ & $119.61(11)$ & $\mathrm{C} 12-\mathrm{C} 11-\mathrm{H} 11$ & 119.9 \\
\hline $\mathrm{C} 4-\mathrm{C} 5-\mathrm{C} 6$ & $119.73(11)$ & $\mathrm{O} 2-\mathrm{C} 12-\mathrm{C} 11$ & $122.68(10)$ \\
\hline $\mathrm{C} 4-\mathrm{C} 5-\mathrm{H} 5$ & 120.14 & $\mathrm{O} 2-\mathrm{C} 12-\mathrm{C} 13$ & $117.95(10)$ \\
\hline $\mathrm{C} 6-\mathrm{C} 5-\mathrm{H} 5$ & 120.14 & $\mathrm{C} 11-\mathrm{C} 12-\mathrm{C} 13$ & $119.35(10)$ \\
\hline $\mathrm{C} 1-\mathrm{C} 6-\mathrm{C} 5$ & $120.91(11)$ & $\mathrm{C} 12-\mathrm{C} 13-\mathrm{C} 14$ & $120.02(11)$ \\
\hline $\mathrm{C} 1-\mathrm{C} 6-\mathrm{H} 6$ & 119.54 & $\mathrm{C} 12-\mathrm{C} 13-\mathrm{H} 13$ & 119.99 \\
\hline $\mathrm{C} 5-\mathrm{C} 6-\mathrm{H} 6$ & 119.54 & $\mathrm{C} 14-\mathrm{C} 13-\mathrm{H} 13$ & 119.99 \\
\hline $\mathrm{O} 1-\mathrm{C} 7-\mathrm{C} 8$ & $107.38(12)$ & $\mathrm{C} 13-\mathrm{C} 14-\mathrm{C} 15$ & $121.12(10)$ \\
\hline $\mathrm{O} 1-\mathrm{C} 7-\mathrm{H} 7 \mathrm{a}$ & 109.47 & $\mathrm{C} 13-\mathrm{C} 14-\mathrm{H} 14$ & 119.44 \\
\hline $\mathrm{O} 1-\mathrm{C} 7-\mathrm{H} 7 \mathrm{~b}$ & 109.47 & $\mathrm{C} 15-\mathrm{C} 14-\mathrm{H} 14$ & 119.44 \\
\hline $\mathrm{C} 8-\mathrm{C} 7-\mathrm{H} 7 \mathrm{a}$ & 109.47 & $\mathrm{C} 9-\mathrm{C} 15-\mathrm{C} 10$ & $122.37(10)$ \\
\hline $\mathrm{C} 8-\mathrm{C} 7-\mathrm{H} 7 \mathrm{~b}$ & 109.47 & $\mathrm{C} 9-\mathrm{C} 15-\mathrm{C} 14$ & $119.18(10)$ \\
\hline $\mathrm{H} 7 \mathrm{a}-\mathrm{C} 7-\mathrm{H} 7 \mathrm{~b}$ & 111.48 & $\mathrm{C} 10-\mathrm{C} 15-\mathrm{C} 14$ & $118.24(10)$ \\
\hline
\end{tabular}




\section{supporting information}

$\begin{array}{llll}\mathrm{C} 4-\mathrm{O} 1-\mathrm{C} 7-\mathrm{C} 8 & -177.51(11) & \mathrm{N} 1-\mathrm{C} 9-\mathrm{C} 15-\mathrm{C} 10 & -15.04(18) \\ \mathrm{C} 9-\mathrm{N} 1-\mathrm{C} 1-\mathrm{C} 2 & -35.10(15) & \mathrm{N} 1-\mathrm{C} 9-\mathrm{C} 15-\mathrm{C} 14 & 170.16(11) \\ \mathrm{C} 9-\mathrm{N} 1-\mathrm{C} 1-\mathrm{C} 6 & 146.67(11) & & \end{array}$

Hydrogen-bond geometry $\left(A,{ }^{\circ}\right)$

\begin{tabular}{lllll}
\hline$D-\mathrm{H} \cdots A$ & $D-\mathrm{H}$ & $\mathrm{H} \cdots A$ & $D \cdots A$ & $D-\mathrm{H} \cdots A$ \\
\hline $\mathrm{C} 7-\mathrm{H} 7 b \cdots \mathrm{O} 2^{\mathrm{i}}$ & 0.96 & 2.50 & $3.3393(15)$ & 147 \\
$\mathrm{O} 2-\mathrm{H} 2 o \cdots \mathrm{N} 1^{\mathrm{ii}}$ & $0.894(17)$ & $1.825(17)$ & $2.7098(12)$ & $170.0(14)$
\end{tabular}

Symmetry codes: (i) $x-1 / 2, y+1,-z+3 / 2$; (ii) $-x+1, y-1 / 2,-z+3 / 2$. 